\title{
Discrimination of stroke-related mild cognitive impairment and vascular dementia using EEG signal analysis
}

\begin{abstract}
Stroke survivors are more prone to developing cognitive impairment and dementia. Dementia detection is a challenge for supporting personalized healthcare. This study analyzes the electroencephalogram (EEG) background activity of 5 vascular dementia $(\mathrm{VaD})$ patients, 15 stroke-related patients with mild cognitive impairment (MCI), and 15 control healthy subjects during a working memory (WM) task. The objective of this study is twofold. First, it aims to enhance the discrimination of $\mathrm{VaD}$, stroke-related MCI patients, and control subjects using fuzzy neighborhood preserving analysis with QR-decomposition (FNPAQR); second, it aims to extract and investigate the spectral features that characterize the post-stroke dementia patients compared to the control subjects. Nineteen channels were recorded and analyzed using the independent component analysis and wavelet analysis (ICA-WT) denoising technique. Using ANOVA, linear spectral power including relative powers $(R P)$ and power ratio were calculated to test whether the EEG dominant frequencies were slowed down in $\mathrm{VaD}$ and stroke-related MCI patients. Non-linear features including permutation entropy (PerEn) and fractal dimension (FD) were used to test the degree of irregularity and complexity, which was significantly lower in patients with $\mathrm{VaD}$ and stroke-related MCI than that in control subjects (ANOVA; $p<0.05$ ). This study is the first to use fuzzy neighborhood preserving analysis with QR-decomposition (FNPAQR) dimensionality reduction technique with EEG background activity of dementia patients. The impairment of post-stroke patients was detected using support vector machine (SVM) and $k$-nearest neighbors $(k N N)$ classifiers. A comparative study has been performed to check the effectiveness of using FNPAQR dimensionality reduction technique with the SVM and $k$ NN classifiers. FNPAQR with SVM and $k \mathrm{NN}$ obtained 91.48 and $89.63 \%$ accuracy, respectively, whereas without using the FNPAQR exhibited 70 and $67.78 \%$ accuracy for SVM and $k N N$, respectively, in classifying $\mathrm{VaD}$, stroke-related MCI, and control patients, respectively. Therefore, EEG could be a reliable index for inspecting concise markers that are sensitive to $\mathrm{VaD}$ and stroke-related MCI patients compared to control healthy subjects.
\end{abstract}

Keyword: Electroencephalography; ICA-WT; Relative power; Permutation entropy; Fractal dimension; Vascular dementia; Mild cognitive impairment 